Let us consider an instrument capable of measuring flux at two different frequencies, $\nu_{1}$ and $\nu_{2}$. Using our cosmological model we may calculate how $F_{\nu_{1}}$ and $F_{\nu,}$ vary with the source redshift. The redshift itself is not observable, but we can make a 'colour-luminosity' diagram: $\Delta \log F_{\nu_{2}} / F_{\nu_{1}}$ against $\Delta \log F_{\nu_{1}}$, where the differences are given with respect to a fiducial source at $z=0.2$. Examples of such colour-luminosity relations are shown in Figure 2 for a few choices of the two spectral parameters, $\alpha$ and $\alpha_{1}$, and for $\log \nu_{2} / \nu_{1}=0.5$. The trend is always the same: the weaker sources have softer spectra. This is so because the spectra were assumed to be of the type given by equation (1), in which the first derivative of the slope is negative. The softening of the spectra is more noticeable when the frequency baseline $\nu_{2} / \nu_{1}$ is large, and the spectral index varies rapidly ( $\alpha_{1}$ is large). In fact, if the hardness of the spectrum is defined as $\log \left(F_{2} / F_{1}\right)$, then its variation with the source redshift is proportional to $\alpha_{1} \times \log \left(\nu_{2} / \nu_{1}\right)$. The larger the spectral baseline, the larger the 'colour' change due to redshift.

The larger the limiting redshift for the sample, the stronger the 'colour' change. The BATSE is the first experiment that is sensitive enough to see the 'edge' of the distribution; that is, to reach $z \sim 1$, assuming that the cosmological model provides the correct interpretation of the 'edge'. The spectra of BATSE sources are therefore the first that may show the expected correlation between the spectral hardness and the burst intensity.

The real bursters are not likely to be standard candles, and their spectra are observed to have a large range of slopes ${ }^{7}$, for the strong as well as for the weak bursts. Therefore, instead of a single line, as in Fig. 2, a lot of scatter is expected, and the trend can only be detected statistically. This correlation seems to be present in the spectra of BATSE bursts, with the weaker bursts being somewhat softer ${ }^{5}$. It is difficult to judge at present whether the effect is real. If confirmed, this correlation would provide a spectral indication that the weak bursts are redshifted with respect to the strong ones. The effect may be detectable even without absolute calibration of the spectra. Once the spectra are well calibrated, and the values of $\alpha$ and $\alpha_{1}$ are measured, it will be possible to estimate the redshift of the sources using the empirical equivalents of Fig. 2.

If the weakest BATSE bursts are at a redshift $z \approx 1.7$, then their light curves (time histories) should be stretched in time by a factor $(1+z)$ as compared with the strongest bursts. It is well known that burst durations cover many orders of magnitude, so it is not clear at all if a relatively small factor like 2.7 is detectable. A small subset of 'simple' single-peaked bursts as observed by BATSE ${ }^{6}$ does, however, show the expected correlation: the rise times of the weakest bursts are longer, on average, than the rise times of the strongest bursts. It is too early to claim that this is the redshift effect. All possible instrumental effects have to be understood first. If confirmed, the correlation between rise time and peak intensity will indicate that the bursters are at cosmological distances.

Received 26 November 1991; accepted 6 January 1992

1. Fishman, G. J. et at. presented at Second Gamma Ray Observatory Workshop. 23-25 September (Annapolis, Maryland, 1991)

2. Meegan, C. A. et al. IAU Circ. No. 5358 (1991); Nature 355, 143-145 (1992).

3. Paczyniski, B. Acta Astr. 41, 257-267 (1991).

4. Mao. S. \& Paczyński, B. Astrophys. J. (submitted).

5. Paciesas, W. et al. presented at Huntsville Gamma-Ray Burst Workshop, 16-18 October (Huntsville Alabama, 1991.

6. Kouveliotou, C. et al. presented at Huntsville Gamma-Ray Burst Workshop, 16-18 October (Huntsville, Alabama, 1991)

7. Weinberg, S. Gravitation and Cosmology (Wiley, New York, 1991)

8. Higdon, J. C. \& Lingenfelter. R. E. Ann. Rev. Astr. Astrophys. 28, 401-436 (1990).

ACKNOWLEDGEMENTS. This project was supported by the NSF and NASA

\section{Gamma-ray bursts in the galactic halo}

\section{J. J. Brainerd}

Space Science Laboratory, ES-65, NASA/Marshall Space Flight Center, Huntsville, Alabama 35812, USA

THE angular and luminosity distributions of the $\gamma$-ray bursts observed by the BATSE instrument, on the Gamma Ray Observatory satellite, cannot be explained by sources confined to the galactic plane'. Instead the observations are consistent with a nearly isotropic source density that falls with distance. Although this permits a cosmological origin, it also permits an origin in the halo of our Galaxy, preserving some aspects of models in which galactic neutron stars are the sites of the bursts. I show here that significant isotropy can be achieved with a spherically symmetric halo model if it extends out beyond $100 \mathrm{kpc}$. Large halo core radii enhance isotropy, although consistency with observation is possible for core radii as small as $\mathbf{5} \mathrm{kpc}$ if the halo radius is sufficiently large. The intrinsic luminosity distribution of $\gamma$-ray bursts must be treated as a free parameter to fit the observations. If $\gamma$-ray bursts are from the halo, they are likely to be old population II neutron stars, because models based on pulsars escaping from the galactic plane have strong anisotropies.

The leading model for $\gamma$-ray bursts is the emission of radiation in the magnetosphere of a neutron $\operatorname{star}^{2}$. Their rapid rise time and $10-\mathrm{ms}$ variability suggest a source smaller than $10^{8} \mathrm{~cm}$, and the numerous bursts with fluxes above $10^{-5} \mathrm{erg} \mathrm{cm}^{-2} \mathrm{~s}^{-1}$ give luminosities of order $10^{38} \mathrm{erg} \mathrm{s}^{-1}$ at $300 \mathrm{pc}$, the luminosities associated with accreting neutron stars. Additional support for this model comes from the observation of spectral features in the Konus data that can be interpreted as redshifted electron- positron annihilation lines and cyclotron resonances from a magnetic field of $\sim 4 \times 10^{12} \mathrm{G}$ (ref. 3 ). Ginga provided stronger evidence by observing in three separate bursts two spectral features interpreted as the first two cyclotron resonances ${ }^{4-6}$. Assuming a galactic origin, many astrophysicists expected a concentration of burst sources along the galactic plane similar to the spatial distributions of X-ray binaries and radio pulsars.

The BATSE instrument of the Gamma Ray Observatory (GRO) satellite is admirably suited to find the distribution of $\gamma$-ray bursts on the $\mathrm{kky}^{7}$. The preliminary results, based on 117 $\gamma$-ray bursts, indicate that the sources are isotropic to the statistical error of a random distribution ${ }^{1}$. The average cosine of the

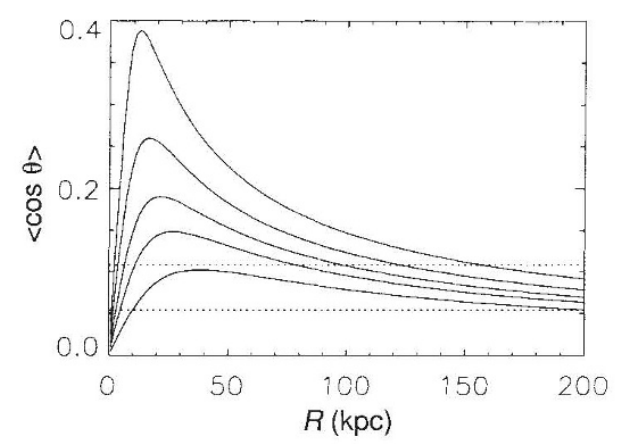

FIG. 1 Plot of $\langle\cos \theta\rangle$, the average cosine of the burst angle relative to the Galactic Centre for the galactic halo model, as a function of the maximum observable distance $R$. The curves, from top to bottom, are for halo core radii of $4.25,8.5,12.75,17$ and $25.5 \mathrm{kpc}$. The dotted lines mark $\langle\cos \theta\rangle=$ 0.054 and 0.108 , at one and two standard deviations from zero for the current observations. 


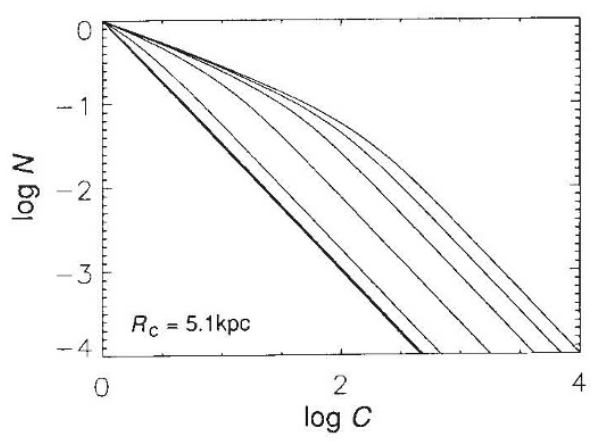

FIG. 2 Plot of $\log N$ against $\log C$. The number of bursts producing a maximum count rate greater than $C$ is plotted for a core radius of $5 \mathrm{kpc}$ for the galactic halo. The units are arbitrary. From top to bottom, the values of the maximum observable distance $R$ are $170,127.5,85,42.5,17,8.5$ and $4.25 \mathrm{kpc}$, with the last two curves almost identical.

angle between the source and the Galactic Centre is $\langle\cos \theta\rangle=$ $0.008 \pm 0.054$ (the published standard deviation ${ }^{1}$ of 0.071 is in error; G. J. Fishman, personal communication). There is no evidence of either the galactic plane or of the Galactic Centre. These results show that the weak bursts are as isotropic as the previously observed strong bursts ${ }^{8,9}$. In addition, the average ratio of the volume delineated by a source (that is, enclosed within a sphere extending to that source) to the maximum volume observed is $\left\langle V / V_{\max }\right\rangle=0.34 \pm 0.03$ (ref. 1), which is smaller than the $0.419 \pm 0.18$ found by the Signe experiment ${ }^{10}$. If the sources were uniformly distributed through space, one would expect 0.5 ; that the observed value falls below this suggests that the source density decreases with distance. The conclusion forced on us if we assume a non-Solar System origin is that these objects are either in an extended galactic halo ${ }^{11}$ or are at cosmological distances ${ }^{12}$. Below I show that the present results cannot exclude a halo origin, although the observations place tight constraints on the allowable models.

The galaxy has a dark halo extending to $50 \mathrm{kpc}$ and, if the interpretation of the magellanic stream is correct, extending beyond $100 \mathrm{kpc}$ (ref. 13). Models for the halo generally assume a mass distribution of $\rho \propto\left(r_{\mathrm{c}}^{2}+r^{2}\right)^{-1}$, where $r_{\mathrm{c}}$ is the halo core radius, for a radial distance from the Galactic Centre of $r<r_{1}$, where $r_{1}$ is the outer radius of the halo. The mass density drops to zero for $r>r_{1}$. Assuming that the peak fiux from $\gamma$-ray bursts drops below background before $r$ approaches $r_{1}$, and that the sources are distributed as the halo mass, one finds that for $r_{\mathrm{c}}=5 \mathrm{kpc}$, the sources are highly skewed toward the Galactic Centre if the maximum observable distance $R$ is $\sim 10 \mathrm{kpc}$, but are relatively isotropic if $R$ exceeds $50 \mathrm{kpc}$ (ref. 12). As $r_{\mathrm{c}}$ increases, the bursts become more isotropic for all $R$. Figure 1 shows $\langle\cos \theta\rangle$ as a function of maximum observable distance for several values of $r_{\mathrm{c}}$. This calculation assumes that $\gamma$-ray bursts have only one peak luminosity. The value $\langle\cos \theta\rangle=0.1$ is within two standard deviations of the BATSE result. This value is consistent with core radii of $8.5 \mathrm{kpc}$ and greater in Fig. 1 for $R>100 \mathrm{kpc}$, and is consistent with $r_{\mathrm{c}}=4.25 \mathrm{kpc}$ for $R>150 \mathrm{kpc}$. The galactic-halo model gives $\langle\cos \theta\rangle \geqslant 0.05$, and is therefore invalid if a statistical sample of bursts has $\langle\cos \theta\rangle=0$ with a standard deviation much less than 0.05 .

The $r^{-2}$ dependence of the halo beyond the core radius $r_{\mathrm{c}}$ leads to $\mathrm{d} \log N / \mathrm{d} \log C=-\frac{1}{2}$, where $C$ is a photon count rate and $N$ is the number of $\gamma$-ray bursts with a peak photon count rate above $C$. If the $\gamma$-ray sources are located nearby so that their distances are much less than the halo length scale, one finds $\mathrm{d} \log N / \mathrm{d} \log C=-\frac{3}{2}$. Figure 2 shows $\log N$ against $\log C$ for a uniform-luminosity burst distribution with maximum observable distances $R$ of $4.25,8.5,17,42.5,85,127.5$ and
$170 \mathrm{kpc}$. For $R<17 \mathrm{kpc}, \mathrm{d} \log N / \mathrm{d} \log C \approx-\frac{3}{2}$, but for $R>$ $42.5 \mathrm{kpc}, \mathrm{d} \log N / \mathrm{d} \log C$ is $-\frac{1}{2}$ for the weakest bursts, and breaks to $-\frac{3}{2}$ as the burst strength increases. These larger distances therefore produce a relatively isotropic distribution with - a gradient $\mathrm{d} \log N / \mathrm{d} \log C$ that deviates from $-\frac{3}{2}$. A multi-luminosity burst population can further smooth the break between $-\frac{1}{2}$ and $-\frac{3}{2}$ at the cost of a larger $\langle\cos \theta\rangle$, so $\mathrm{d} \log N / \mathrm{d} \log C$ is a measure of both the spatial distribution and the peak luminosity distribution of $\gamma$-ray bursts. Equivalently, the value of $\left\langle V / V_{\text {max }}\right\rangle$ must fall in a range between $\frac{1}{4}$ and $\frac{1}{2}$, with the precise value dependent on the peak luminosity distribution. Allowing for a range of luminosities lowers Paczyński's restriction of $r_{\mathrm{c}}>17 \mathrm{kpc}$ (ref. 12) on the size of the galactic halo's core. A maximum count-rate distribution that is a $C^{-0.7}$ power law up to a cut-off value $C_{\text {co }}$ gives the observed value of $\left\langle V / V_{\max }\right\rangle \approx 0.35$ while simultaneously giving $\langle\cos \theta\rangle \leqslant$ 0.1 for $R>200 \mathrm{kpc}$ and $r_{\mathrm{c}} \geqslant 8.5 \mathrm{kpc}$. For $\langle\cos \theta\rangle<0.15$, one can have $\left\langle V / V_{\max }\right\rangle \approx 0.35$ for $R>100 \mathrm{kpc}$ and $r_{\mathrm{c}} \leqslant 5.0 \mathrm{kpc}$. This is shown in Fig. 3, where the solid lines are contours of constant $R$ and the dotted lines are contours of constant $r_{c}$. The plotted region shifts to the right as the power-law index $\delta$ of the count rate distribution function $C^{-\delta}$ decreases. A similar shift occurs if the source distribution falls less rapidly than $r^{-2}$. The BATSE results are at present consistent with a galactic-halo origin of $\gamma$-ray bursts for reasonable values of the galactic core radius. But with a $2-3 \sigma$ deviation from the observed value, the constraints on the model are tight. A test of the galactic-halo model is evident from Fig. 3: the value of $\langle\cos \theta\rangle$ against $\left\langle V / V_{\max }\right\rangle$ is strongly dependent on the maximum observable distance, which is equivalent to a dependence on the minimum count rate in the observations.

The galactic-halo model places an upper limit on the burst luminosity. BATSE can detect $\gamma$-ray burst fluxes down to $10^{-7} \mathrm{erg} \mathrm{cm}^{-2} \mathrm{~s}^{-1}$. For a burst to fall below the background inside a $200-\mathrm{kpc}$ galactic halo, the peak burst luminosity must be less than $4 \times 10^{41} \mathrm{erg} \mathrm{s}^{-1}$. A similar number can be derived for $\gamma$-ray bursts from the halo of nearby spiral galaxies. For M31, the luminosity must be greater than $2 \times 10^{42} \mathrm{erg} \mathrm{s}^{-1}$ to be seen as a burst excess over a circle of radius $15^{\circ}$ (ref. 14). This suggests that the peak luminosity function must drop by at least an order of magnitude between these two luminosities.

The shape of the halo is unknown, so the most conservative approach is to assume that halo neutron stars have an isotropic distribution about the Galactic Centre and that our offset from

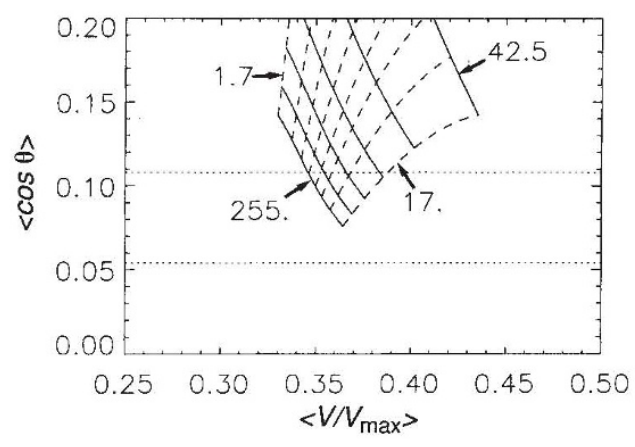

FIG. 3 Plot of $\langle\cos \theta\rangle$ against $\left\langle V / V_{\max }\right\rangle$. The relationship between these two values for different halo core radii and maximum observable burst distances is plotted for a power-law burst count rate of index 0.7 . The solid lines are calculated for variable core radius and fixed values of maximum observable distance; from upper right to lower left the maximum observable distances are $42.5,85,127.5,170,212.5$ and $255 \mathrm{kpc}$, all assuming a solar distance from the Galactic Centre of $8.5 \mathrm{kpc}$. The dashed curves from the top left curve to the bottom right curve are caiculated for constant galactic halo core radii of $1.7,3.4,5.1,6.8,8.5,10.2,12.75$ and $17 \mathrm{kpc}$. The dotted curves represent one and two standard deviations away from $\langle\cos \theta\rangle=0$ of the BATSE resuits. 
the Galactic Centre is the only source of anisotropy. Although physically unjustified, this model produces the minimum likely value of the dipole moment relative to the Galactic Centre, as any asymmetry in the galactic halo will add to the size of the dipole moment unless one assumes a fortuitous cancellation of the offset dipole by the asymmetry. The halo neutron stars may be the remnants of population II stars. A second possibility is that neutron stars are ejected into the halo from the galactic disk at a high velocity, as is indicated by proper motion and interstellar scintillation studies ${ }^{15,16}$, which find velocities averaging $\sim 150 \mathrm{~km} \mathrm{~s}^{-1}$ and ranging beyond $400 \mathrm{~km} \mathrm{~s}^{-1}$ relative to the galactic disk velocity, and is suggested by the high-velocity interpretation ${ }^{17}$ of pulsar PSR1757-24. In such a model, the comparison of the spatial distribution of $\gamma$-ray bursts to the distribution of pulsars is invalid, because the pulsar distribution is determined by the short pulsar lifetime of $10^{7} \mathrm{yr}$ (ref. 18). If a significant number of neutron stars are injected below the escape velocity, which is likely from current observations, the distribution function will be quite different from the assumed dark halo distribution, producing considerable dipole and quadrupole moments ${ }^{19}$. If most injected neutron stars have velocities far above the escape velocity of the galactic halo, in which case the catalogues of pulsar velocities are grossly incomplete, then these stars will have a spatial distribution that falls roughly as $r^{-2}$ far from the galactic disk and an asymmetric core enveloping the galactic disk. Reliance on neutron stars in the galactic plane requires extreme models for populating the galactic halo.
Received 7 October; accepted 26 December 1991.

A. A. al. TAU CIRC $5358(1991)$

. Phys. Rep. 206, 327-394 (1991)

Mazets, E. P. et al. Astrophys. Space Sci. 80, 3-83 (1982

Murakami, T. et al. Nature 335, 234-235 (1988)

5. Fenimore, E. E. et al. Astrophys. J. 335, L71-74 (1988)

6. Yoshida. A., Murakami, T., Nishimura, J., Kondo. I. \& Fenimore. E. E. Publs astr. Soc. Japan 43 (in the press)

7. Fishman, G. J. et al. Proc. GRo Science Workshop. 2.39-2.44 (Goddard Space Flight Center Greenbelt, Maryland, 1989)

8. Hartmann, D. \& Blumenthal, G. R. Astrophys. J. 342, 521-526 (1989)

9. Hartmann, D. \& Epstein, R. I. Astrophys, J 346, 960-966 (1989).

10. Atteia, J. L. et al. Nature 351, 296-298 (1991)

11. Jennings, M. C. High Energy Transients in Astrophysics (ed. Woosley, S. E.), 412-421 (American Institute of Physics, New York, 1984)

12. Paczyński, B. Acta Astr. 41, 157-167 (1991)

13. Binney, J. \& Tremaine, S. Galactic Dynamics (Princeton University Press, 1987)

14. Atteia, J. L. \& Hurley, K. Adv. Space Res. 6, 39-43 (1986).

15. Lyne, A. G., Anderson, B. \& Salter. M. J. Mon. Not. R. astr. Soc. 201, 503-520 (1982).

16. Cordes, J. M. Astrophys. J. 311, 183-196 (1986).

17. Frail D. A. \& Kulkarni, S. R. Nature 352,785 787(

18. Tayior. J. H. \& Stinebring, O. R. A. Rev. Astr. Astrophys. 24, 285-327 (1986)

19. Paczynski, B. Astrophys. J. 348, 485-494 (1990)

ACKNOWLEDGEMENTS. This work was done while J.J.B. was an NRC research associate at the NASA-Marshall Space Flight Center.

\section{Collimation of astrophysical jets by inertial confinement}

\author{
Vincent Icke*, Garrelt Mellema*, Bruce Balick $\dagger$, \\ Frits Eulderink* $*_{\ddagger}$ \& Adam Frank $\dagger$
}

* Sterrewacht Leiden, Postbus 9513, 2300 RA Leiden, The Netherlands

† Department of Astronomy, University of Washington, Seattle,

Washington 98195, USA

† Shell Research Laboratories, Postbus 3003, 1003 AA Amsterdam,

The Netherlands

MANY astrophysical objects, from young stars, Herbig-Haro objects and planetary nebulae up to active galactic nuclei, can be very simply modelled as isotropic sources of high-energy tenuous gas embedded in dense toroidal clouds. Here we describe numerical simulations showing how such an arrangement can in general circumstances give rise to a well collimated jet, as is observed in many of these systems. Our model is a two-dimensional generalization of the interacting-winds description of planetary nebulae. Where the two winds come into contact, a discontinuity is formed, which is dragged out by the fast outflowing gas into a chimney along the polar axis. High-energy gas rushes up this channel and flows out around the top, creating a hot backflow which keeps the chimney in place. The inner shock, enclosing the source of the fast wind, also aids in collimation, and ionization cones such as those observed in active galactic nuclei may also form.

In our work on the hydrodynamics of aspherical planetary nebulae (PNe), we have come across a mechanism for the formation of hydrodynamic jets which is very effective, and which easily extends to situations other than PNe. Planetary nebulae are bubbles blown by a tenuous, fast stellar wind into a dense, slow, fossil red-giant envelope. Balick ${ }^{1}$ generalized this model to two dimensions to explain the morphologies of aspherical PNe. Observations indicate that the envelope has cylindrical symmetry, and that the density is higher at the equator than at the poles. The PN morphology and its evolution is then purely a consequence of the mass distribution in the envelope and its interaction with the fast stellar wind.

Analytical $^{2-5}$ and numerical ${ }^{6-8}$ studies of the hydrodynamics of this model have yielded results that comfortably fit the many morphologies of PNe. Here we argue that the model has broader astrophysical applications.

The details of our hydrodynamic method ${ }^{6,8}$ have been discussed elsewhere. We use spherical coordinates $(r, \theta, \phi)$ with $\partial / \partial \phi=0$. As initial conditions in the envelope, we took the family of disk-toroids described $b^{3-5}$

$$
\begin{gathered}
\rho(\theta, r)=\frac{\rho_{*}}{A(\theta)}\left(\frac{r_{0}}{r}\right)^{2} \\
A(\theta) \equiv 1-\alpha\left(\frac{\mathrm{e}^{\beta \cos 2 \theta-\beta}-1}{\mathrm{e}^{-2 \beta}-1}\right)
\end{gathered}
$$

Here $\alpha$ describes the equator-to-pole contrast ('e/p ratio'), and $\beta$ governs the decrease of the density with polar angle $\theta$. This function represents plausible models of gaseous disks. Its isodensity contours range from ellipsoidal $(\beta \rightarrow 1)$ through quasi-toroidal $(\beta>3, \alpha \rightarrow \infty)$ to annular $(\beta \approx 1, \alpha>20)$. Narrow funnels can also be constructed $(\beta>10, \alpha>5)$.

We have run more than 50 simulations. Of these, we show one case which is representative for the jet formation mechanism we discovered. The initial conditions are as follows (all quantities are given at a reference position on the symmetry axis, $\theta=0$, radius $r_{0}=1.6 \times 10^{14} \mathrm{~m}=0.005 \mathrm{pc}$ ). The envelope wind has a mass density of $\rho_{*}=3 \times 10^{-17} \mathrm{~kg} \mathrm{~m}^{-3}$ and a radial velocity of $10 \mathrm{~km} \mathrm{~s}^{-1}$. The tangential velocity is zero. The temperature is $T^{*}=1,000 \mathrm{~K}$; choosing $50<T^{*}<10,000 \mathrm{~K}$ produces similar results. The asphericity is $\alpha=0.9$, producing an e/p ratio of 10 . The steepness parameter $\beta=6$.

The spherical inner wind has a density $\rho_{\mathrm{c}}=10^{-20} \mathrm{~kg} \mathrm{~m}^{-3}$ and a velocity $v_{\mathrm{c}}=2,500 \mathrm{~km} \mathrm{~s}^{-1}$. The density contrast between inner and outer winds is then 3,000 . The temperature of the fast wind is $T_{\mathrm{c}}=50,000 \mathrm{~K}$, so the Mach number is $\mathcal{M}=112$. Four frames of the basic hydrodynamic variables are shown in Fig. 1, at $1.4 \times 10^{10} \mathrm{~s}$ after the onset of the fast wind.

A cool tongue-like deformation extends from the contact discontinuity, forming a chimney along the symmetry axis and helping to collimate the outflow. Turbulent hot gas streams back from the head of the outflow; this exerts a further confining pressure. Finally, the inner 'reverse' shock forms a prolate barrel shape along the symmetry axis, as expected ${ }^{3,9}$. The fast gas around $\theta \approx 40^{\circ}$ passes obliquely through this shock, and is thereby deflected upward. The outer layers of the chimney 